\title{
Continuing mind for primary care medicine as total family care mailing list (TFC-ML) group
}

\begin{abstract}
There were historically two great doctors for primary care (PC) medicine in Japan. They are Dr. Shigeaki Hinohara and Dr. Yoshikazu Tasaka. Tasaka was always active in medical treatment, organizational management, postgraduate education, and information dissemination using the Internet, and started Total Family Care Mailing List (TFC-ML) in 1998. TFC-ML included medical information with his comments every day for long. Even after his death in 2007, TFC-ML activity has been continued by many voluntary PC physicians. His TFC mind has been transmitted to future PC physicians. His inspiration may often come to TFC members for better total family care.
\end{abstract}

Keywords: Yoshikazu Tasaka, Total Family Care Mailing List (TFC-ML), Japan Primary Care Association (JPCA), Japan Society of Family Medicine (JSFM), Shigeaki Hinohara

\author{
Volume 4 Issue 5 - 2020 \\ Hiroshi Bando, 1,2 Shigekiyo Nakanishi,, ${ }^{1,3}$ \\ Toshinobu Kuji, ${ }^{1,4}$ Yoshihisa Matsumura, ${ }^{1,5}$ \\ Toru Yokoi,' Tomoyuki Kido,' Fusako \\ Nemoto,' Keiko Hayano,' Yasushi Fujiwara,' \\ Takakazu Matsuki,' Hideki Yasuda' \\ 'Total Family Care Mailing List (TFC-ML) group, Japan \\ ${ }^{2}$ Tokushima University / Medical research, Tokushima, Japan \\ ${ }^{3}$ Nakanishi Clinic, Hiroshima, Japan \\ ${ }^{4}$ Kuji Clinic, Ehime, Japan \\ ${ }^{5}$ Matsumura Clinic, Nara, Japan
}

Correspondence: Hiroshi Bando, MD, PhD, FACP,Tokushima University /Medical Research

Address: Nakashowa I-61, Tokushima 770-0943, Japan,

Tel +8I-90-3 I87-2485, Email pianomd@bronze.ocn.ne.jp

Received: August 24, 2020 | Published: September 30, 2020

\section{Commentary}

Primary health care and primary medical care are necessary for the people to live everyday in good health worldwide. Primary care (PC) medicine has been born and developed according to the situation of each country. Japanese PC includes remarkably high quality and contains the 5 factors of ACCCC (Access to Care, Comprehensive Care, Coordination of Care, Continuity of Care, Contextual Care) by Saultz. ${ }^{1}$ The first author aspired to PC medicine, studied in a US residency program, and in 2017 presided over the 8th National Convention of the Japan Primary Care Association (JPCA). ${ }^{2}$ There are historically two great doctors who have been involved in PC in Japan. One is Dr. Shigeaki Hinohara (1911-2017), the father of Japanese PC medicine. ${ }^{3,4} \mathrm{He}$ has brought a new concept and dimension to Japan such as health education, problem-oriented system (POS), nursing education, PC medicine, music therapy, and so on. ${ }^{5}$ Another is Dr. Yoshikazu Tasaka. He was a super doctor always active in medical treatment, organizational management, postgraduate education, and information dissemination using the Internet, and was known and respected to everyone in the field of PC medicine ${ }^{6}$ (Figure 1). In this article, his achievements would be introduced associated with probable suggestions for the future development of PC medicine across the world.

Dr. Tasaka graduated from Kawasaki Medical School in 1981. Post-graduate trainings were performed in the pathology and general medicine departments, as well as residency training of family medicine in Minnesota, USA for 1 year. He was involved in the establishment as the director of the family clinic affiliated with medical university in 1994. Dr. Tasaka had started Total Family Care Mailing List (TFC-ML) as the director in 1998. ${ }^{6}$ Since 2000, he has been a board of the Japan Society of Family Medicine (JSFM), and subsequently served as a director. He has continued extremely busy working with family practice, activity of Hiroshima Medical
Association and management of TFC-ML every day. Unfortunately, he was dead suddenly in February 2007 at the age of 49. Dr. Tasaka had majored in pathophysiology, internal medicine, primary care medicine and infective diseases. As to infection region, he presented the research on alveolar macrophages. ${ }^{7}$ Even after his death, his coworkers had kept the research. As to upper respiratory tract infection (URTI), Prospective cohort observational study was performed for primary care clinics in Japan. ${ }^{8}$ As a result, 691 non-influenza cases showed nonspecific URTI $80 \%$, acute pharyngitis $13 \%$ and acute bronchitis $5 \%$. Antibiotic use will be hopefully adequate adhering to the guideline proposed by the American College of Physicians. ${ }^{9}$

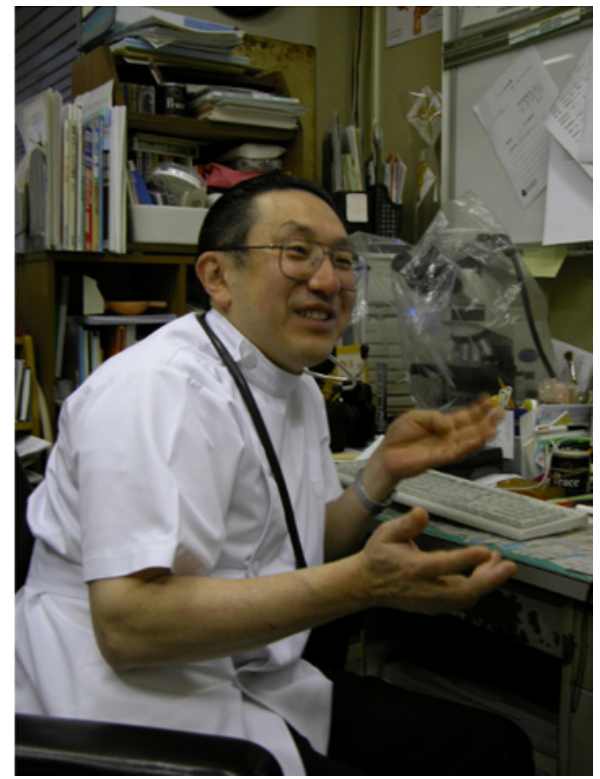

Figure I Dr.Tasaka at his desk with his favorite microscope. 
In the primary care setting, loxoprofen has been often given for URTI. There was a double-blind, randomized, placebo-controlled trial in 23 facilities of Japan. As a result, loxoprofen did not influence the recovery process significantly. ${ }^{10}$ This research had been continued for long by Great Cold Investigators-II. Consequently, Dr. Tasaka's research mind for infectious and respiratory diseases was transmitted to many physicians for long. Dr. Tasaka continued his projects on undergraduate and postgraduate clinical research in the area of medical education. ${ }^{11,12} \mathrm{He}$ also expanded his research into multi-center study of nationwide survey. ${ }^{13,14}$ Regarding these results, the achievements were widely reported through papers, academic conferences, mailing lists, and others. The research results over the years have been presented by the collaborators (Figure 2).

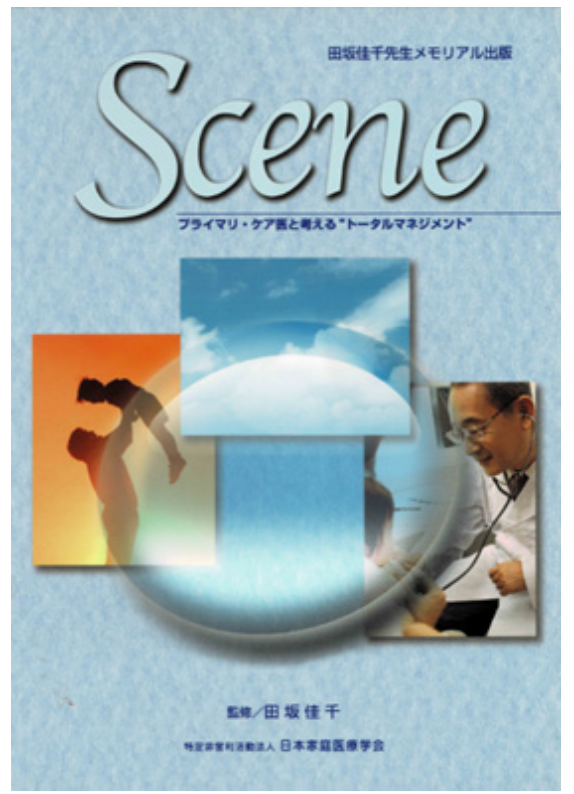

Figure 2 Textbook "Scene": Total management to consider with primary care physician. It was published by Japan Society of Family Medicine (JSFM) as the memorial book of Dr. Tasaka, in which all content was edited by Dr. Tasaka.

He started working in his family clinic in 1997 and also activity in the community medical associations. Since 1998, he had started transmitting medical information using the Internet. This included a unique method in which Dr. Tasaka had added each comment to posted emails. ML members were mainly from the JSFM. His comments were excellent, and the points of the problems were clearly organized, and then everyone was encouraged to attend free discussions. As regards to TFC-ML, its usefulness and issues was discussed, and showed that a role of moderator, excess of information and correspondence with slander would be important. ${ }^{14}$ With continuous practice and evaluation, the number of members gradually increased to about 2500. Everyone looked forward to TFC-ML, learned various medical tips, and felt impressed with its quality.

Dr. Tasaka had continued working hard all day, and providing useful information for long years. He had often commented "I am just immature trainee for life", and practiced humbly learning and teaching. It was admirable. When he stayed at Hokkaido in February, 2007 , he was suddenly dead with acute distress at the age of 49 , which was unbelievable news for people. ${ }^{15}$ Even if he is not present, many physicians have maintained his TFC mind in their hearts. In order to leave his TFC mind for future generations, the TFC secretariat was set up immediately and continued to operate TFC-ML by several board members. The number of all members was 2,841 (August 7, 2020), and family doctors, specialists and medical students have participated in live discussions freely as before. It is also a significant opportunity to deepen mutual understanding between practitioners and hospital doctors. ${ }^{16}$ As to the board members, Drs. Nakanishi, Kuji, Yokoi, Matsumura have mainly dealt with the core matters of TFC-ML for long.

Several TFC related projects have kept going until now. Tasaka's memorial lecture has been held every year associated with significant presentations. Furthermore, the Tasaka award was established in JPCA, which is similar to Hinohara award. In every annual congress of JPCA, the Tasaka award is provided to the physician who has contributed much to the community. ${ }^{17}$ The activity of TFC-ML has been continuing by many members more than 20 years. We suppose that Dr. Tasaka is always observing our medical practice from heaven. We are unconscious and may not notice his suggestion. In various opportunities, however, Dr. Tasaka often sends us his adequate inspiration, which can contribute to better total family care for a patient just before us.

\section{Conflicts of interest}

None.

\section{Funding}

None.

\section{References}

1. Saultz JW. Textbook of family medicine. McGraw-Hill, Medical professions Division, 2001. 830p.

2. The $8^{\text {th }}$ Annual Conference of Japan Primary Care Association.

3. Hinohara S. A proposal for new term habit disease instead of adult disease. Educ Health Serv. 1978;5:1-2.

4. Doba N, Abe H, Hayashida N, et al. Semi-supervised exercise training program for patients with coronary heart disease - its effectiveness and possible diagnostic implications for predicting their severity. Jpn Circ J. 1983;47:735-743

5. Bando H, Yoshioka A, Iwashimizu Y, et al. Development of Primary Care, Lifestyle Disease and New Elderly Association (NEA) in Japan Common Philosophy With Hinohara-ism. Prim Health Care. 2017;7:281.

6. TFC-ML.

7. Tasaka Y, Sumi M, Niki Y, Soejima R. Rokitamycin uptake by alveolar marcophages. Jap J Antibiotics. 1988;41(7):836-840.

8. Tomii K, Matsumura Y, Maeda K, et al. Minimal Use of Antibiotics for Acute Respiratory Tract Infections: Validity and Patient Satisfaction. Internal Medicine. 2007;46(6), 267-272.

9. Harris AM, Hicks LA, Qaseem A. High Value Care Task Force of the American College of Physicians and for the Centers for Disease Control and Prevention. Appropriate Antibiotic Use for Acute Respiratory Tract Infection in Adults: Advice for High-Value Care From the American College of Physicians and the Centers for Disease Control and Prevention. Ann Intern Med. 2016;164(6):425-434.

10. Goto M, Kawamura T, Shimbo T, et al. Influence of loxoprofen use on recovery from naturally acquired upper respiratory tract infections: a randomized controlled trial. Intern Med. 2007;46(15):1179-1186.

11. Ban N, Tsuda T, Tasaka Y. Patients' Views about Undergraduate Clinical Training: Targeting Improved Clinical Clerkship Training on the Ward. Medical Education. 1994;25(1):35-42. 
12. Takeda Y, Otaki J, Matsumura S, et al. Teaching Ambulatory Care Medicine in Japan: A Nationwide Survey. Medical Education. 2003;34(4):245-249.

13. Matsumura S, Takeda Y, Otaki J. Postgraduate Ambulatory Care Training at Government-accredited Teaching Hospitals: Results of a Nationwide Survey in Japan. Medical Education. 2003;34(5):289-295.

14. Hashimoto N, Matsumura T, Aizawa Y. Promotion of Continuing Medical Education for Physicians by Using the Mailing List. Medical Education. 2003;34(6):363-367.
15. Portrait of a hero: Dr. Yoshikazu Tasaka, offering 2500 doctors challenging job by email every day. Nikkei Medical. 2004;36(4):191-193.

16. Hospitalist division of JPCA.

17. Japan Primary Care Association. 